\title{
Maturidade da gestão do conhecimento: a importância da infraestrutura organizacional para o desenvolvimento dos estágios
}

Giovana Escrivão ${ }^{I}$

http://orcid.org/0000-0002-6348-3199

Sergio Luis da Silva ${ }^{I I}$

http://orcid.org/0000-0002-5970-9386

I Universidade de São Paulo, SP, Brasil.

Doutora em Engenharia de Produção pela Universidade Federal de São Carlos.

II Universidade Federal de São Carlos, SP, Brasil.

Doutor em Engenharia Mecânica São Carlos pela Universidade de São Paulo.

http://dx.doi.org/10.1590/1981-5344/4022

O objetivo da pesquisa é identificar o impacto e descrever o comportamento da infraestrutura organizacional nos estágios de desenvolvimento da gestão do conhecimento. Para tal foi realizado um levantamento de dados com 125 gestores das 46 Unidades da Empresa Brasileira de Pesquisa Agropecuária - Embrapa, empresa de base tecnológica nacionalmente reconhecida devido aos altos índices de inovação. Neste estudo a infraestrutura organizacional necessária ao desenvolvimento da gestão do conhecimento foi definida como: estrutura organizacional, tomada de decisão, processo de comunicação, grau de formalização das atividades e ambiente. Os resultados revelam que a estrutura organizacional se torna mais flexível, a tomada de decisão mais descentralizada, a comunicação em todos os 
sentidos sofre um aumento consecutivo e o ambiente permite maior interação e troca de conhecimento entre as pessoas ao longo do avanço dos estágios, corroborando com a teoria. No entanto, exatamente ao contrário do que sugere a teoria, o grau de formalização das atividades aumenta com o avanço dos estágios. A pesquisa contribui ao complementar o estudo da gestão do conhecimento e ao identificar e descrever, empiricamente pela primeira vez, a variável infraestrutura organizacional nos estágios da gestão do conhecimento.

Palavras-chave: Gestão do conhecimento. Estágios. Maturidade. Infraestrutura organizacional.

\section{Knowledge management maturity: the significance of organizational infrastructure for the development of its stages}

The aim of this paper is to identify the impact and describe the behavior of the organizational infrastructure in the stages of the knowledge management development. For this, a data collection was carried out with 125 managers of the 46 Units of the Brazilian Agricultural Research Company - Embrapa, a TechnologyBased Company, which is national and internationally recognized due to its high innovation rates. In this study, the organizational infrastructure required to develop the knowledge management was defined as: organizational structure, decision-making, communication process, degree of formalization of activities and organizational environment. The results concluded that the organizational structure becomes more flexible, the decision-making more decentralized, the communication process in all directions (top down, bottom up and between areas) increases consecutively and the environment allows greater communication and interaction among people throughout the advancement of 
the stages, corroborating the theory. However, in the opposite direction to what is suggested by the theory, the degree of formalization of activities increases over the development of the stages. The research contributes to complement the study of knowledge management and to identify and describe, empirically for the first time, the organizational structure of knowledge management stages.

Keywords: Knowledge management. Stages. Maturity. Organizational infrastructure.

Recebido em 17.06.2019 Aceito em 23.10.2020

\section{Introdução}

Estudiosos de diversas disciplinas - sociologia, economia, administração, dentre outras - concordam que uma transformação mundial tem ocorrido e que no centro dela está o conhecimento. Como consequência, as organizações têm se concentrado em explorara criação, transferência e uso do conhecimento organizacional de forma mais efetiva (DAVENPORT; DE LONG; BEERS, 1998), consolidando-o como o recurso econômico chave e fonte de vantagem competitiva mais importante para as organizações (DRUCKER, 1993).

Esse contexto tem levado ao surgimento de inúmeras iniciativas por parte das organizações, no entanto, muitos autores concordam que parte dessas iniciativas ocorre de maneira desarticulada e a maioria dos projetos são limitados quanto ao seu impacto; poucos contribuem efetivamente com os resultados organizacionais (DAVENPORT; DE LONG; BEERS, 1998; DE LONG; FAHEY, 2000).

A maturidade da gestão do conhecimento (MGC) surge como uma tentativa de diminuir a distância entre as promessas e os resultados efetivamente alcançado pela gestão do conhecimento (GC). AMGC identifica o estágio em que a organização se encontra e quais as áreas primordiais para que ela se desenvolva até o estágio seguinte (LEE; KIM; YU, 2001; RASULA; VUKSIC; STEMBERGER, 2008).

Os modelos de maturidade são um passo em direção à melhoria da capacidade de desenvolvimento organizacional (GALBRAITH, 1982; DAFT, 2003). A organização que tem conhecimento do seu desenvolvimento consegue: identificar o estágio em que ela se encontra; saber as 
necessidades atuais e futuras e como agir para satisfazê-las;realizar um planejamento coerente com as demandas de cada momento (GALBRAITH; 1982; ADIZES, 1990; DAFT, 2003). No entanto, os modelos de maturidade da gestão do conhecimento (MMGC) ainda apresentam muitas lacunas, nenhum modelo teve sua metodologia totalmente aceita (KULKARNI; ST LOUIS, 2003; SAJEVA; JUCEVICIUS, 2012). A pesquisa sobre o tema ainda é recente - a primeira publicação data de pouco mais de uma década - e ainda apresenta muitas controvérsias e lacunas a serem superadas (LEE; KIM, 2001; FENG, 2005; LIN, 2011).

A principal lacuna diz respeito a falta de critérios sistemáticos e validações empíricas na identificação e inclusão de fatores críticos na construção do MMGC (KRUGER; SNYMAN, 2005; TEAH; PEE; KANKANHALLI， 2006; RASULA; VUKSIC; STEMBERGER, 2008; PEE; KANKANHALLI, 2009; LIN, 2011; SINHA; DATE, 2013). Apesar da literatura sobre GC já ter identificado diversas práticas e fatores críticos, os MMGC não demonstram um consenso (TEAH; PEE; KANKANHALLI, 2006; PEE; KANKANHALLI, 2009; LIN, 2011).O fato de os modelos serem desenvolvidos com base em diferentes fatores dificulta sua comparação, avaliação e aplicação para os pesquisadores e profissionais da área. Para desenvolver MMGC é necessário identificar sistematicamente os fatores que devem compor o MMGC (TEAH; PEE; KANKANHALLI, 2006; PEE; KANKANHALLI, 2009; LIN, 2011).

Nesse sentido esse artigo identificou os fatores críticos ao desenvolvimento da GC e que devem compor um MMGC e analisou um dos fatores - a infraestrutura organizacional - por meio da pesquisa empírica.

A infraestrutura da organização é fator básico para o desenvolvimento da GC, ela pode promover e facilitar as práticas de GC ou, ao contrário, ela pode funcionar como uma barreira, dificultando o desenvolvimento dessas práticas (HEDLUND, 1994; NONAKA; TAKEUCHI, 1995; GRANT, 1996; O'DELL; GRAYSON, 1998; GOLD; MALHOTRA; SEGARS, 2001; CONLEY; ZHENG, 2009).

\section{Revisão teórica}

Maturidade é o processo de desenvolvimento de um objeto ao longo do tempo (KLIMKO, 2001; JIULING; JIANKANG; HONGJIANG, 2012; SERENKO; HULL; BONTIS, 2014) e seus modelos são resultado da aplicação da abordagem do ciclo de vida organizacional (CVO), segundo o qual a organização se desenvolve por meio de estágios ao longo do tempo até chegar a um nível considerado perfeito (GAÁL et al., 2008).

No caso da GC, a maturidade se refere ao estado de efetividade em gerir e alavancar os ativos do conhecimento na organização até o momento em que ela está explicitamente definida, gerida, controlada e 
gerando resultados eficazes para a organização (KULKARNI; ST LOUIS, 2003; TEAH; PEE; KANKANHALLI, 2006; SAJEVA; JUCEVICIUS, 2012).Consequentemente, os MMGC ajudam as organizações avaliarem seu progresso relativo na implementação das práticas de GCao proporcionar uma visão global do processo, destacando os elementos associados a cada estágio e indicando possíveis melhorias (TEAH; PEE; KANKANHALLI, 2006; LIN, 2007; GAÁL et al., 2008; OLIVEIRA; PEDRON; MAÇADA, 2010; LIN, 2011).

A revisão da literatura permitiu a definição dos estágios, conforme apresenta o Quadro 1. O estágio 1 é denominado de "iniciação funcional", pois é o momento que a organização passa a ter consciência das práticas de GC; caracteriza-se pelo uso de ferramentas isoladas no sentido de gerenciar o conhecimento organizacional. O estágio 2 é denominado "especialização funcional", pois é o momento em que a organização formaliza e reconhece a GC como uma área importante à consecução de seus objetivos; o estágio caracteriza-se pelo desenvolvimento da infraestrutura necessária às práticas de GC, como sistemas, suporte, tecnologia; e ocorre a elaboração de estratégia e planejamento da GC. O estágio 3 denominado de "integração interna" caracteriza-se pela institucionalização da GC, isto é, esta passa a um componente da cultura organizacional e é realizado o controle, o monitoramento, a medição e a melhoria contínua das práticas de GC.E, por fim, no estágio 4 as práticas de GC são integradas à rede externa da organização.

Quadro 1 - Estágios da Gestão do Conhecimento

\begin{tabular}{|c|c|c|c|}
\hline ES TÁGIO 1 & ES TÁGIO 2 & ES TÁGIO 3 & ES TÁGIO 4 \\
\hline $\begin{array}{l}\text { INICIAÇÃO } \\
\text { FUNCIONAL } \\
\end{array}$ & $\begin{array}{l}\text { ESPECIALIZAÇÃO } \\
\text { FUNCIONAL } \\
\end{array}$ & $\begin{array}{c}\text { INTEGRAÇÃO } \\
\text { INTERNA } \\
\end{array}$ & $\begin{array}{c}\text { INTEGRAÇÃO } \\
\text { EXTERNA } \\
\end{array}$ \\
\hline Consciência & Formalização & Institucionalização & Rede externa \\
\hline $\begin{array}{l}\text {-Uso de } \\
\text { ferra mentas } \\
\text { isoladas no sentido } \\
\text { de gerenciar o } \\
\text { conhecimento } \\
\text { organizacional. }\end{array}$ & $\begin{array}{l}\text {-Desenvolvimento da } \\
\text { infraestrutura necessária às } \\
\text { práticas de gestão do } \\
\text { conhecimento - sistemas, } \\
\text { suporte, tecnologia. } \\
\text {-Estratégia e planeja mento } \\
\text { da gestão do } \\
\text { conhecimento. }\end{array}$ & $\begin{array}{l}\text {-A gestão do conhecimento } \\
\text { está embutida na cultura } \\
\text { organizacional. } \\
\text {-É realizado o controle, o } \\
\text { monitora mento, a medição } \\
\text { e a me lhoria contínua das } \\
\text { práticas de gestão do } \\
\text { conhecimento. }\end{array}$ & $\begin{array}{l}\text {-Integração das } \\
\text { práticas de gestão do } \\
\text { conhecimento à rede } \\
\text { externa. } \\
\text {-Parcerias. }\end{array}$ \\
\hline
\end{tabular}

Fonte: Própria

A busca sistemática e a revisão da literatura também possibilitaram a análise dos MMGC existes. A maioria dos artigos sobre o tema fazem 
uma revisão parcial da literatura, incluindo apenas alguns poucos MMGC; poucos fazem uma análise mais completa da literatura, com grande inclusão dos modelos presentes na literatura. Alguns modelos se resumem a pesquisa teórica, ou seja, alguns autores fazem uma revisão de alguns MMGC e apenas descrevem os estágios com base em estágios já existentes, não trazendo grande contribuição ao tema. Outras pesquisas se limitam a um diagnóstico de uma ou mais organizações com objetivo único de identificar em que estágio, determinada organização se encontra. Os estudos que buscam testar o modelo proposto apresentam uma seleção de elementos que não aborda todos os fatores amplamente citados pela literatura de GC. Há autores que excluem alguns fatores, para reduzir o número de variáveis ou para excluir fatores complexos ou de difícil mensuração, o que torna os modelos incompletos. Apesar de muitos fatores se repetirem nos MMGC, cada autor seleciona um conjunto diferente de fatores, sem critérios ou justificativa de seleção e algumas vezes excluem algum fator amplamente citado e já consolidado pela literatura, como a cultura organizacional, por exemplo. Portanto, apesar de alguns fatores serem citados por mais de um autor, a análise comparativa dos modelos demonstra que ainda não há um consenso sobre eles.

Dessa maneira, falta uma seleção sistemática dos fatores que devem compor um MMGC, bem como uma validação empírica que os confirme (LEE; KIM; YU, 2001; LEE; CHOI, 2003; TEAH; PEE; KANKANHALLI, 2006; KANKANHALLI, 2009; LIN, 2011).

Assim, essa pesquisa realizou uma busca sistemática e uma revisão da literatura de GC e dos MMGC com objetivo de identificar os fatores críticos de sucesso (FCS) da GC e que devem compor um MMGC. Após o cruzamento e a contagem dos fatores citados em ambas literaturas (GC e MMGC), foram identificados os fatores que devem compor um MMGC, conforme demonstra o Quadro 2.

Quadro 2 - Definição dos FCS da GC

\begin{tabular}{ll}
\hline \hline \multicolumn{1}{c}{ FCS } & \multicolumn{1}{c}{ FCS PARA A GC } \\
\hline \hline & \\
& -Estrutura organizacional fle xível \\
Infraestrutura & -Tomada de decisão descentralizada \\
organizacional & -Proces so de comunicação em todas as direções \\
& -Baixo grau de formalização das atividades e processos \\
& -Ambiente que favorece a interação \\
& \\
Tecnologia & -Ferramentas tecnológicas de processamento \\
& -Ferramentas tecnológicas integradas entre si \\
& -Ferramentas tecnológicas que permitem a interação entre as pessoas
\end{tabular}


Cultura

Gestão de recursos humanos

Apoio da alta gerência
-Co mportamentos que demonstrem colaboração e aprendizage m

-Tre inamento

-Recompensas

-Oportunidade de participação

-Coordenação

-Motivação

Fonte: Própria

Feito isto, para construir um MMGC, o comportamento de cada fator deve ser analisado nos diferentes estágios da GC. Esse artigo analisa o comportamento da variável infraestrutura organizacional.

A GC só ocorre se houver uma infraestrutura básica a favor desta tarefa.

Nesse sentido, a estrutura organizacional pode estimular ou inibir as interações entre as pessoas e consequentemente facilitar ou dificultar as práticas de GC (HEDLUND, 1994; NONAKA; TAKEUCHI, 1995; GRANT, 1996; O'DELL; GRAYSON, 1998; GOLD; MALHOTRA; SEGARS, 2001; CONLEY; ZHENG, 2009). Em essência, é importante que as estruturas organizacionais sejam flexíveis promovendo o intercâmbio e a colaboração além das fronteiras internas e externas da organização (GOLD; MALHOTRA; SEGARS, 2001). A burocracia e a rigidez dos sistemas de conhecimento funcionam como uma barreira à sua gestão, em casos extremos, toda a organização torna-se um plano, onde nada pode ser mudado, pois como tudo está conectado, a mudança se torna algo mais difícil. Assim, a flexibilidade, através da transferência e rotação das pessoas, por exemplo, é importante para que possam ocorrer mudanças e adaptações. Ainda nesse sentido, os grupos de pessoas que são fixos por meio da estrutura hierárquica dificultam a interação (HEDLUND, 1994). "More formalized and centralized structure dampens KM success whereas a flexible informal structure facilitates it" ${ }^{11}$ (CONLEY; ZHENG, 2009, p.339).

O formato dos processos decisórios também faz parte da infraestrutura organizacional que facilita ou dificulta a GC. A concentração do processo decisório reduz soluções criativas, enquanto que a dispersão do poder facilita a espontaneidade e a experimentação. Além disso, a estrutura centralizada dificulta a comunicação interdepartamental e o compartilhamento frequente de ideias. Sem um fluxo constante de

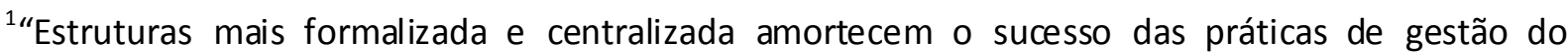
conhecimento, enquanto uma estrutura flexível e informal facilita o bom desempenho destas."
} 
comunicação e ideias, a criação do conhecimento não ocorre (TEECE, 2000; LEE; CHOI, 2003). Centralização refere-se ao local de autoridade, decisão e controle dentro de uma entidade organizacional. A estrutura organizacional descentralizada facilita um ambiente onde os funcionários participam no processo de construção do conhecimento mais espontaneamente. Ambientes de trabalho participativo fomentam a criação de conhecimento, motivando envolvimento dos membros organizacionais. Portanto, a diminuição centralização pode levar a um aumento da criação de conhecimento (TEECE, 2000).

A comunicação em todos os níveis é essencial para que a GC ocorra. Isso implica em um foco menos voltado para o topo da corporação e mais voltado para os níveis intermediários, já que o conhecimento está cada vez mais disperso, devido à rápida mudança tecnológica e educacional. A comunicação exclusivamente vertical também limita a interação. Para interação, multi e pluridisciplinar, faz-se necessário a comunicação vertical e horizontal. Além disso, a comunicação middle-up-down também propicia maior interação se comparada as comunicações top-down e bottom-up, já que o fluxo de informações flui nas duas direções (HEDLUND, 1994).

Vários estudos têm chegado à conclusão de que a formalização enfraquece a GC, mas, em contraste, o estudo de Lee e Choi (2003) reflete dois aspectos diferentes de formalização. Formalização refere-se ao grau em que as decisões e relações de trabalho são regidas por regras formais, políticas e procedimentos. Ela pode inibir a formalização de atividades relacionadas ao conhecimento tácito, tais como socialização e externalização, mas pode incentivar atividades relacionadas ao conhecimento explícito, tais como combinação e internalização. De qualquer forma, a criação do conhecimento requer flexibilidade e menos ênfase nas normas de trabalho. As novas ideias parecem ser restringidas quando regras formais dominam uma organização. A flexibilidade pode acomodar maneiras melhores de fazer as coisas, resultando no aumento da criação de conhecimento, já que esta requer variação a fim de ser mais adaptáveis quando surgem problemas imprevistos. Formalidade sufoca a comunicação e a interação necessárias para criar conhecimento (LEE; CHOI, 2003).

De nada adianta garantir todas essas condições se o ambiente não favorece aspectos relacionados a interação. A GC envolve o estabelecimento de um ambiente propício à criação de conhecimento mais eficaz, a sua transferência e seu uso (NONAKA; TAKEUCHI, 1995; DAVENPORT; DE LONG; BEERS, 1998). A organização do espaço físico deve proporcionar um ambiente (contexto capacitante) que facilite a interação, a comunicação e a troca de conhecimento entre as pessoas (DAVENPORT; PRUSAK, 2003).Ao fornecer um campo alargado de interação entre os membros da organização para estes partilharem ideias 
e perspectivas e estabelecerem diálogos, a organização pode permitir que as pessoas cheguem a novos insights e/ou mais interpretações de suas próprias informações e conhecimentos. É nesse espaço de compartilhamento na organização que as relações emergem, possibilitando esse compartilhamento de tempo e espaço através da experiência direta, formando um contexto de linguagem comum entre os participantes do processo (NONAKA;TAKEUCHI, 1995; NONAKA; TOYAMA, 2003). Esse contexto organizacional pode ser físico (um escritório, uma sala, um espaço para negócios), virtual (teleconferência, e-mail) e/ou mental (ideias, experiências compartilhadas) e é partilhado por dois ou mais indivíduos na organização (NONAKA; TAKEUCHI, 1995; NONAKA; KONO, 1998).

Assim, para fornecer infraestrutura organizacional para desenvolver as práticas de GC a empresa deve ter uma estrutura flexível, que permita colaboração além das fronteiras formais da organização e movimentações das equipes com pessoas de diferentes áreas participando dos projetos e atividades; processos decisórios descentralizados com participação de todos na organização; comunicação em todos os níveis organizacionais fluindo em todos os sentidos; baixo grau de formalização das atividades permitindo maior flexibilidade na realização destas que permita a GC explícitos e; um ambiente que favoreça e incentive a comunicação, a interação entre as pessoas e o compartilhamento de conhecimento.

\section{Procedimentos da pesquisa}

A busca sistemática, a revisão teórica e a operacionalização das variáveis orientaram a formulação do instrumento de coleta de dados. Após desenvolvida sua primeira versão, o questionário foi submetido a procedimentos que buscam garantir validade de conteúdo, validade técnica e validade empírica do instrumento. Para tal, o questionário foi avaliado por especialistas da área de GC (professores e pesquisadores da Universidade Federal de São Carlos - UFSCar, Universidade de São Paulo USP e Salem State University - SSU, Estados Unidos) garantindo a validade de conteúdo e; especialistas da área de estatística (professores e pesquisadores da Universidade Federal de São Carlos - UFSCar e Universidade de São Paulo - USP e profissionais de uma empresa privada multinacional de material de escritório e da Confederação Nacional da Indústria - CNI) garantindo a validade técnica do instrumento.

$\mathrm{Na}$ tentativa de garantir a validade empírica foram realizados dois pré-testes. O primeiro foi realizado com funcionários do Departamento de TI da Salem State University - SSU, que estava implementando um projeto de GC; de forma que, alguns funcionários tinham contato mais próximo com tais práticas e outros não. Assim, as características desses respondentes são similares ao que se esperava da população do survey - 
organizações mais e menos envolvidas com a GC. Com uma taxa de retorno de 33,3\%, obteve-se 10 respostas dentre os 30 respondentes. Como o questionário desenvolvido possui escalas de variados pontos e variáveis quantitativas categóricas em escala de razão, o Alfa de Cronbach foi calculado considerando as questões que possuem escala de 4 pontos. O valor gerado, 0.89, revela um bom índice de confiabilidade e coerência entre as questões formuladas. Após as devidas correções, um segundo pré-teste foi realizado com alunos do curso de especialização do Programa de Pós-Graduação em Engenharia da Produção da Universidade de São Paulo - USP. Todos os alunos trabalhavam em cargos de gestão de variadas empresas, cargo semelhante aos cargos dos respondentes da survey. Foram obtidas 27 respostas de $80,33,7 \%$ de retorno.

Os dois estudos-piloto foram importantes para o refinamento das questões. O questionário envolve questões que possibilitam identificar o estágio em que cada organização se encontra e questões a respeito do comportamento da variável infraestrutura. No que diz respeito a variável os respondentes foram questionados quanto a rigidez da estrutura organizacional, a concentração da tomada de decisão, a predominância do processo de comunicação, ao grau de formalização das atividades e a caracterização do ambiente.

Os dados da pesquisa principal foram coletados em empresas de base tecnológica, pois elas são caracterizadas pelo uso intensivo de recursos tecnológicos, foco do presente trabalho. Elas são caracterizadas pela presença de pesquisadores de alta qualificação, pelo investimento em pesquisa e desenvolvimento (P\&D) para inovação dos seus produtos (BAÊTA, 1999) e por influenciar parceiros, clientes, fornecedores e concorrentes por meio de uma cultura de inovação (JUGEND; SILVA, 2010). Assim, nessas empresas, o conhecimento organizacional é um recurso essencial e estratégico de geração de vantagem competitiva (COLOMBO; DELMASTRO, 2002; SANTOS; AMATO NETO, 2008; GOMES FILHO, 2010).

As a knowledge and technology intensive industry, innovative enterprises much more depend on knowledge management than the traditional enterprises. Thus, it is one crucial aspect of innovative enterprises' core competitiveness to build the evaluation model of innovative enterprise $\mathrm{KMM}$ and to transform innovations into business performance (FENGJU, CHIAOJING, 2001, p.741). ${ }^{2}$

\footnotetext{
${ }^{2}$ Como uma indústria intensiva em conhecimento e tecnologia, empresas inovadoras dependem muito mais do conhecimento que empresas tradicionais. Assim, esse é um aspecto crucial para a competitividade de empresas inovadoras para o desenvolvimento de um modelo de evolução da
} 
Sendo o conhecimento um recurso estratégico de grande importância para essas empresas, a presente pesquisa teve como objeto de estudo uma EBT reconhecida no que diz respeito à inovação e desenvolvimento de novas tecnologias - a Empresa Brasileira de Pesquisa Agropecuária (Embrapa).

A Embrapa é uma empresa de pesquisa, desenvolvimento e inovação que busca prover novos conhecimentos traduzidos em produtos, processos e serviços para o setor agropecuário. Sua missão é viabilizar soluções para o desenvolvimento sustentável do agronegócio no Brasil por meio da P\&D de novas tecnologias e conhecimento (EMBRAPA, 2014).

São vários os indicativos do nível de inovação da Embrapa. Ela ficou em $1^{\text {a }}$ colocada no levantamento realizado em 2011 pelo Instituto Nacional de Propriedade Intelectual - INPI sobre as instituições de pesquisa brasileiras com mais pedidos de patente nos 18 anos anteriores (INPI, 2011). E, apesar da empresa trabalhar com ciência aplicada, que não tem como objetivo final a publicação de artigos acadêmicos, entre 2006 e 2010 foram publicados 5.073 artigos em periódicos indexados da Web of Science pelos pesquisadores da empresa; e entre o período de 1977 a 2006 o índice H (Hirsch) da mesma foi definido como 56 e média de citação dos artigos de 6,03 (PENTEADO FILHO; AVILA, 2009a; PENTEADO FILHO; AVILA, 2009b).

A Embrapa é uma organização estrategicamente voltada para a P\&D; atua como líder nacional em geração de conhecimento, novas tecnologias e inovação; possui diversas unidades localizadas em diferentes regiões do país, apresentando variações quanto as práticas de GC (diferentes estágios).

A população do estudo são todos os cargos de gerência e chefia (portanto todos que possuíam uma visão ampla da organização que dirigem) das 46 Unidades Descentralizadas da Embrapa, totalizando uma população de 180 respondentes. A amostra foi calculada baseando-se nos seguintes parâmetros: nível de confiança de $95 \%$, erro amostral de $5 \%$ e população de 180 respondentes, resultando em uma amostra de 123 respondentes. Foram obtidas 125 respostas, o que corresponde a uma taxa de retorno de $67,7 \%$ e garante um nível de confiança maior que $95 \%$ e um erro amostral menor que 5\%. Essa taxa de retorno é considerada alta, já que as surveys online na área de gestão alcançam uma taxa de retorno entre $25 \%$ e $35 \%$ (BARUCH; HOLTON, 2008; MARCONI; LAKATOS, 2011).

maturidade da gestão do conhecimento da empresa inovadora e transformar as inovações no desempenho do negócio. 
Nenhuma Unidade foi identificada pelo questionário como pertencente ao estágio 4. Dessa forma, o comportamento da variável infraestrutura foi analisado nos estágios 1, 2 e 3 da GC.

Para analisar os dados coletados foram utilizadas as seguintes técnicas estatísticas: análise descritiva uni e bivariada e teste exato de Fischer.

\section{Resultados}

A infraestrutura da organização pode favorecer ou dificultar o desenvolvimento da GC até sua maturidade dependendo de como se comportam: a estrutura organizacional, o processo de tomada de decisão, o processo de comunicação, o grau de formalização das atividades e o ambiente organizacional. Todos esses fatores foram investigados com auxílio do questionário.

\section{a. Estrutura organizacional}

De acordo com a literatura, uma estrutura organizacional mais flexível favorece as práticas de GC, enquanto uma estrutura mais rígida funciona como uma barreira a elas, dificultando que mudanças ocorram, a troca de informação e conhecimento e a criação de novos conhecimentos. Dessa forma, era esperado que a estrutura organizacional das organizações estudadas se tornassem mais flexíveis com o avanço dos estágios da GC.

O Gráfico 1revela que as características "predominantemente flexível" e "totalmente flexível" nos três estágios estudados são 39\%, 28\% e $52 \%$, respectivamente, revelando uma queda de $11 \%$ na flexibilidade das Unidades estudadas, seguida de um aumento de $24 \%$. Dessa forma, o estágio 2 pode ser caracterizado como o mais rígido dos três e o estágio 3 como o mais flexível.

Gráfico 1 - Estrutura organizacional nos estágios da GC

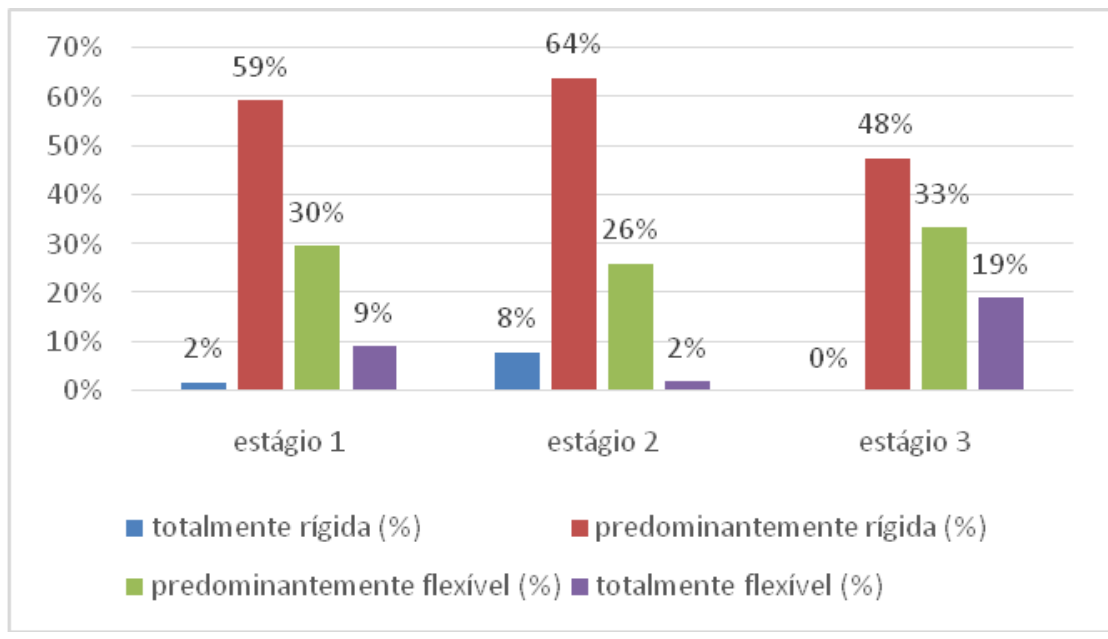




\section{b. Tomada de decisão}

Segundo a teoria, a tomada de decisão centralizada dificulta a disseminação e a GC e a descentralização da tomada de decisão propicia suas práticas.

De acordo com o Gráfico 2, tomada de decisão "totalmente descentralizada" ou "predominante descentralizada" vai de $31 \%$ (estágio 1), para $40 \%$ (2) e $47 \%$ (3). Esse comportamento sugere que com o avanço dos estágios a tomada de decisão tende a ser mais descentralizada.

Gráfico 2 - Tomada de decisão nos três estágios da GC

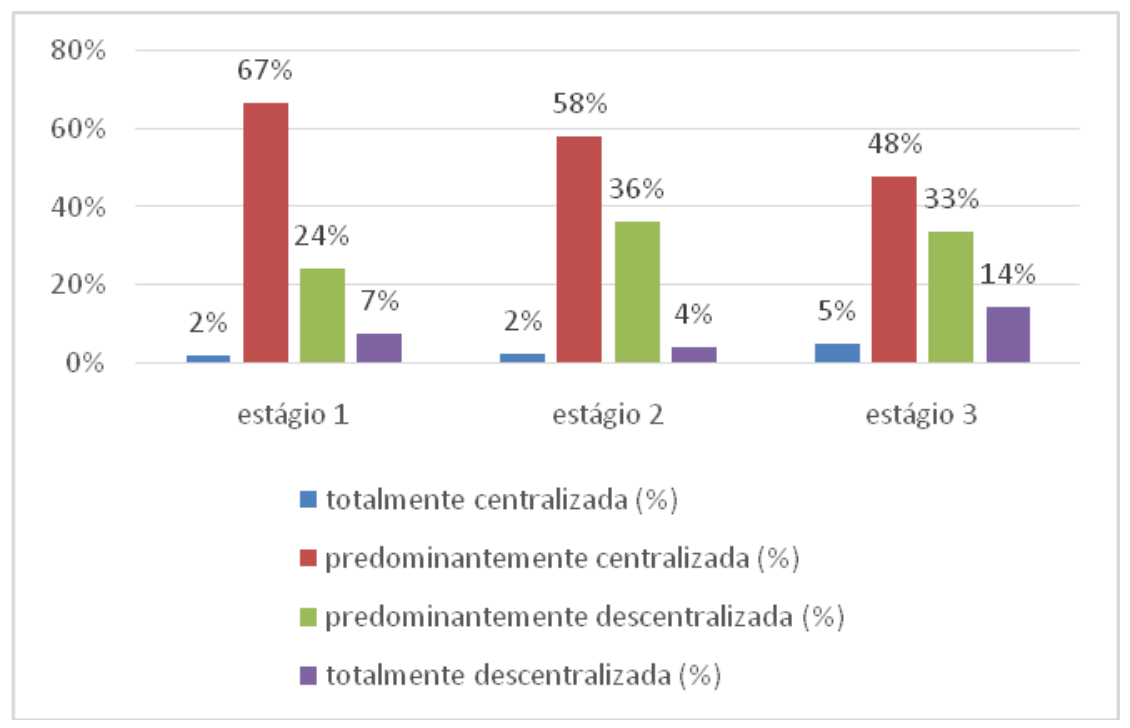

\section{c. Processo de comunicação}

Segundo a teoria, o processo de comunicação flui por meio de três diferentes formas nas organizações: de cima para baixo, de baixo para cima e em todos os sentidos. A comunicação que facilita a ocorrência das práticas de GC é aquela que ocorre em todos os sentidos.

O Gráfico 3 revela a predominância do tipo de comunicação em todos os sentidos (aquela que facilita a GC) nos três estágios estudados, representando um aumento de $17 \%$ no estágio 2 e uma redução pequena de $3 \%$ no estágio 3 . Portanto, podemos afirmar que os estágio 2 e 3 possuem um processo de comunicação mais favorável a GC, sugerindo o crescimento desse tipo de comunicação.

Gráfico 3 - Processo de comunicação nos estágios da GC 


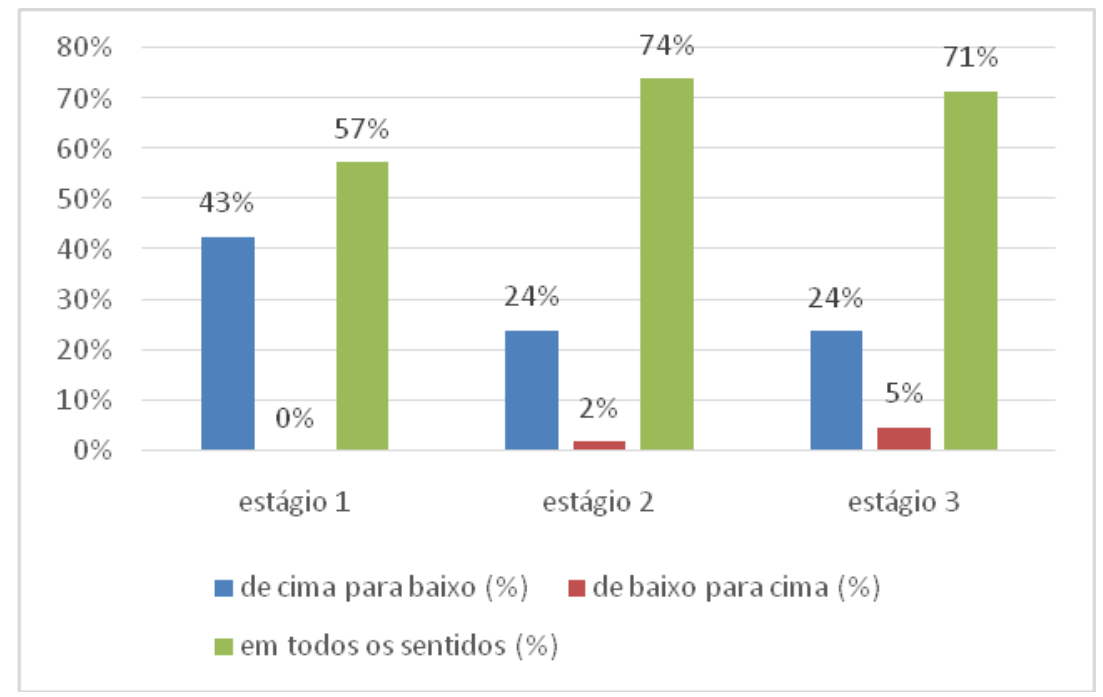

d. Grau de formalização das atividades

O grau de formalização das atividades também influencia o desenvolvimento da GC. O ideal é que o grau de formalização das atividades seja baixo, proporcionando maior flexibilidade à realização das práticas de GC.

O Gráfico 4mostra que o grau de formalização "totalmente baixo" e "predominantemente baixo" são $24 \%, 12 \%$ e $5 \%$, respectivamente nos estágios 1,2 e 3 . Esse resultado sugere um aumento do grau de formalização das atividades. Nota-se ainda que todos os estágios possuem alto grau de formalização predominando.

Gráfico 4 - Grau de formalização das atividades nos estágios da GC 


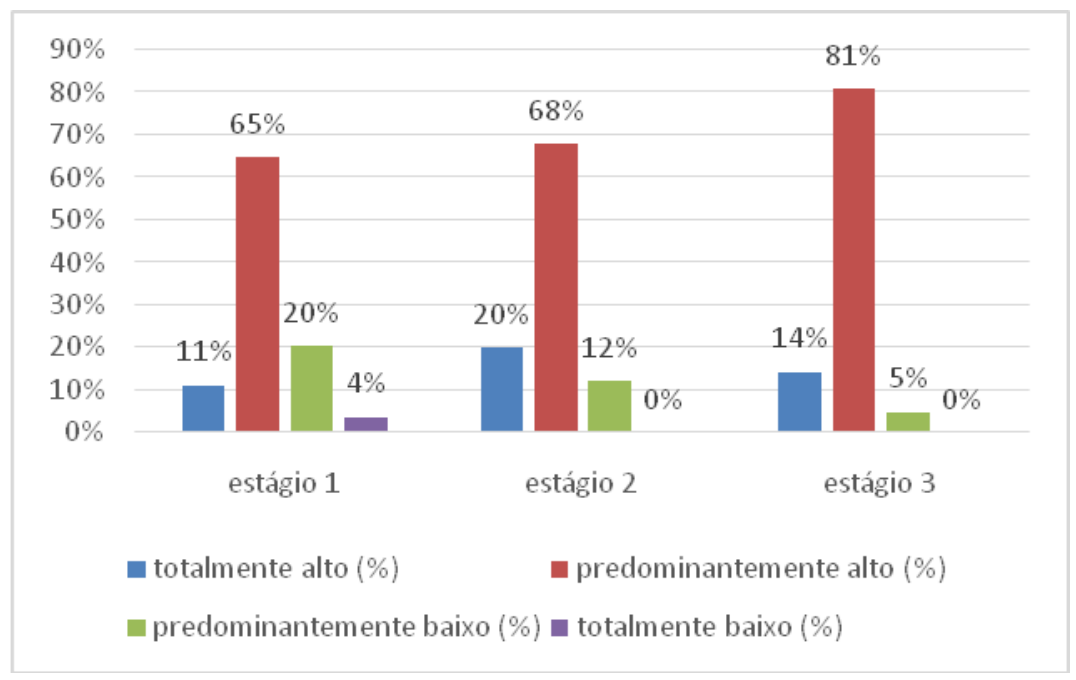

\section{e. Ambiente organizacional}

A literatura afirma que um ambiente favorável à GC deve favorecer a interação entre as pessoas na organização. $O$ ambiente que dificulta que a interação entre os funcionários ocorra age como uma barreira a disseminação de informação e troca de conhecimento e consequentemente dificulta o desenvolvimento da GC.

Quanto ao ambiente organizacional, o Gráfico 5 demonstra que as chefias que acreditam que o ambiente físico que favorece a interação entre as pessoas cresce entre os estágios de $52 \%$ (estágio1), para $60 \%$ (2) e $76 \%$ (3), sugerindo que o ambiente não propício à interação entre os funcionários é progressivamente substituído pelo ambiente que facilita a interação entre as pessoas e a disseminação de conhecimento. Além disso, nota-se que a grande mudança de comportamento não ocorre nos estágios iniciais, mas no estágio 3. 
Gráfico 5 - Ambiente organizacional nos estágios da GC

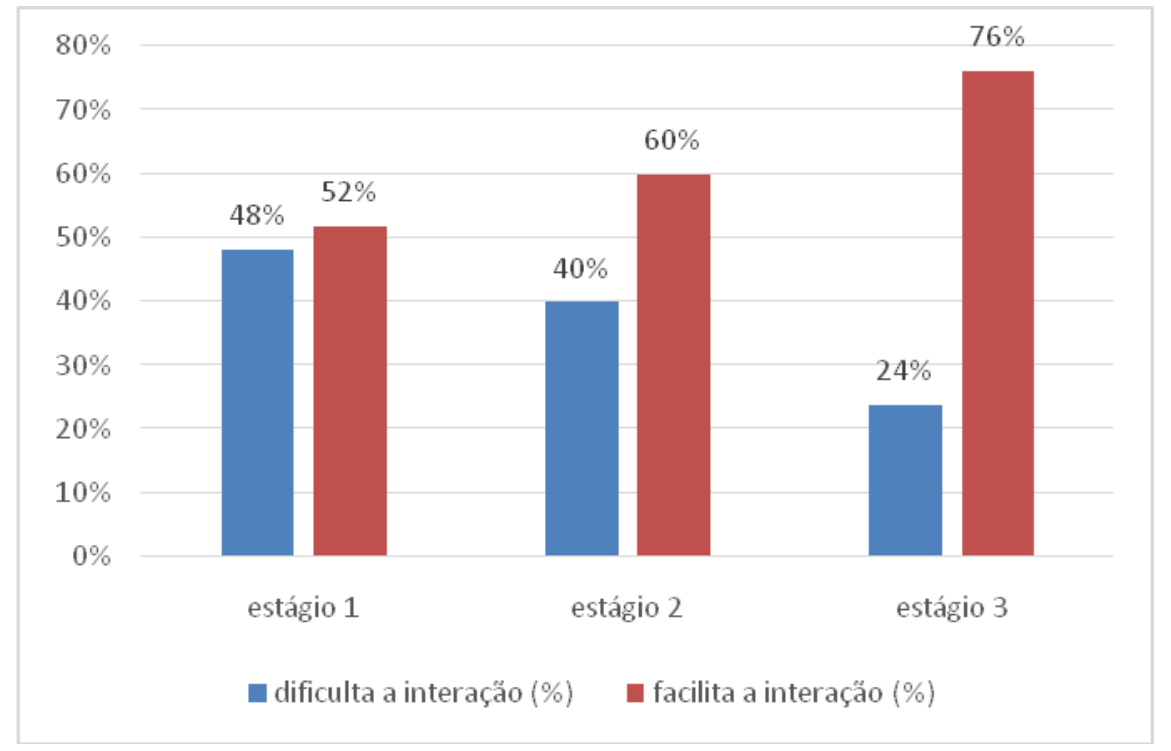

Sintetizando, no estágio 1 a estrutura organizacional apresenta uma caracterização intermediária no quesito rigidez/flexibilidade se comparada aos estágios 2 (mais rígido) e 3 (mais flexível). A tomada de decisão se apresenta mais centralizada que nos estágios seguintes. A comunicação ocorre em todos os sentidos. O grau de formalização das atividades é o mais baixo. E o ambiente físico facilita a disseminação de conhecimento e a interação entre as pessoas, mas em menor grau que nos próximos estágios. No estágio 2 a estrutura organizacional se torna mais rígida que no estágio anterior, sendo inclusive a mais rígida dos três estágios. A tomada de decisão se torna mais flexível e a comunicação e o ambiente se tornam mais favorável à gestão do conhecimento, com aumento considerável da comunicação em todos os sentidos. O grau de formalização aumenta. E no estágio 3 a estrutura se torna mais flexível que no estágio anterior e é, inclusive, a mais flexível dos três estágios. A tomada de decisão continua se tornando mais descentralizada. A comunicação em todos os sentidos se mantém praticamente estabilizada em relação ao estágio 2 . O grau de formalização das atividades aumenta. E o ambiente se torna mais favorável interação.

O Quadro 3 resume a caracterização dos estágios a partir da média das respostas.

Quadro 3- Comportamento da infraestrutura nos estágios da GC

\begin{tabular}{|c|c|c|c|c|}
\hline VARIÁVEL & $\begin{array}{c}\text { SUB- } \\
\text { VARIÁVEL } \\
\end{array}$ & ESTÁGIO 1 & ES TÁGIO 2 & ES TÁGIO 3 \\
\hline $\begin{array}{l}\text { Infra- } \\
\text { estrutura }\end{array}$ & $\begin{array}{c}\text { Es trutura } \\
\text { organizacional }\end{array}$ & $\begin{array}{l}\text {-Estrutura tende } \\
\text { para rígida }\end{array}$ & $\begin{array}{l}\text {-Estrutura se torna } \\
\text { mais rígida }\end{array}$ & $\begin{array}{l}\text {-Estrutura se torna } \\
\text { mais flexível }\end{array}$ \\
\hline
\end{tabular}




\begin{tabular}{|c|c|c|c|}
\hline $\begin{array}{l}\text { Tomada de } \\
\text { decisão }\end{array}$ & $\begin{array}{l}\text {-Tomada de } \\
\text { decisão tende a } \\
\text { centralizada }\end{array}$ & $\begin{array}{l}\text {-Tomada de } \\
\text { decisão se torna } \\
\text { mais } \\
\text { descentralizada }\end{array}$ & $\begin{array}{l}\text {-Tomada de } \\
\text { decisão se torna } \\
\text { mais } \\
\text { descentralizada }\end{array}$ \\
\hline Comunic ação & $\begin{array}{l}\text {-Co municação em } \\
\text { todos os sentidos } \\
\text { predomina }\end{array}$ & $\begin{array}{l}\text {-Comunicação } \\
\text { em todos os } \\
\text { sentidos aumenta }\end{array}$ & $\begin{array}{l}\text {-Comunicação em } \\
\text { todos os sentidos } \\
\text { se estabiliza }\end{array}$ \\
\hline $\begin{array}{r}\text { For malização } \\
\text { das ati vi dades }\end{array}$ & $\begin{array}{l}\text {-Grau de } \\
\text { formalização das } \\
\text { atividades alto }\end{array}$ & $\begin{array}{lr}\text {-Grau } & \text { de } \\
\text { formalização } & \text { das } \\
\text { atividades } & \text { se } \\
\text { torna mais alto } & \end{array}$ & $\begin{array}{lr}\text {-Grau } & \text { de } \\
\text { formalização das } \\
\text { atividades } & \text { se } \\
\text { torna mais alto } & \end{array}$ \\
\hline Ambiente & $\begin{array}{l}\text {-Ambiente } \\
\text { favorável a GC }\end{array}$ & $\begin{array}{l}\text {-Aumenta } \\
\text { ambiente } \\
\text { favorável a GC }\end{array}$ & $\begin{array}{l}\text {-Aumenta } \\
\text { consideravelmen } \\
\text { te o ambiente } \\
\text { favorável a GC }\end{array}$ \\
\hline
\end{tabular}

$\mathrm{Na}$ análise inferencial dos dados, de acordo com os resultados do Teste de Fischer sintetizados na Tabela 1, não há indício de associação entre a estrutura organizacional e os estágios da GC; bem como a tomada de decisão predominante na organização, o grau de formalização e o ambiente também não demonstram existência de associação com os estágios da GC. No entanto, os resultados sugerem existência de associação entre o processo de comunicação e os estágios da GC.

Tabela 1 - Resultados do teste de Fischer

\begin{tabular}{llc}
\hline \hline \multicolumn{1}{c}{ Variável } & \multicolumn{1}{c}{ Sub-variável } & p-value \\
\hline \hline & Estrutura organizacional & 0.1415 \\
Infraestrutura & Tomada de decisão & 0.4319 \\
Organizacional & Comunicação & 0.0936 \\
& Formalização das atividades & 0.3417 \\
& Ambiente & 0.1536 \\
\hline \hline
\end{tabular}

\section{Discussão}

A estrutura organizacional pode estimular ou inibir as interações entre as pessoas e consequentemente facilitar ou dificultar a GC. A estrutura flexível promove o intercâmbio e a colaboração além das fronteiras internas e externas da organização (HEDLUND, 1994; GOLD; MALHOTRA; SEGARS, 2001; CONLEY; ZHENG, 2009). O processo decisório também pode facilitar ou dificultar a GC. A tomada de decisão 
descentralizada proporciona um ambiente no qual os funcionários participam do processo de construção do conhecimento, proporcionando envolvimento dos funcionários e fomentando a participação e a criação de conhecimento (HEDLUND, 1994; TEECE, 2000).

Os resultados da investigação revelam que a estrutura organizacional se torna mais rígida no estágio 2 e mais flexível no estágio 3. Acredita-se que esse fato possa ter relação com os estágios da seguinte maneira: estágio 2 é caracterizado pela formalização da GC e, consequentemente, um aumento das normas e regras que diminuem a flexibilidade; quando no estágio 3 , a gestão do conhecimento já está formalizada e passa a fazer parte da cultura da organização, a qual envolve, além de normas e regras, comportamentos e costumes, que possibilitam maior flexibilidade.

Assim, 61\% dos dirigentes afirmaram que sua Unidade tem rigidez estrutural no estágio 1 de iniciação da GC, presume-se que é devido à finalidade da organização investigada: reconhecidamente desenvolvedora de tecnologia, o que, conceitualmente, exige flexibilidade na definição das funções, da supervisão, acompanhamento por metas. Os dados empíricos revelam ainda o estágio de formalização (estágio 2) como o de mais alto grau de rigidez (72\%); teoricamente compreensível, pois, a formalização exige o estabelecimento de normas, procedimentos e regimentos para a função de GC. Embora geralmente se tenha uma visão da formalização como algo burocrático, como apresenta a teoria da criação do conhecimento, na realidade essa visão cotidiana distorcida do conceito de formalização não é o que ocorreu neste estudo. No estágio 3, foram $48 \%$ dos dirigentes a reconhecer que a estrutura de sua Unidade é rígida, o mais baixo grau de rigidez e maior de flexibilidade; também compreensível, pois a passagem da formalização para a institucionalização significa a mudança da autoridade das normas para o reforço das crenças e valores da cultura organizacional. As pessoas tendem a ver como "mais natural" e menos rígido a regulação por valores do que por normas.

E a tomada de decisão apresenta uma crescente descentralização. Os testes não indicaram associação entre essas variáveis e os estágios da GC. Dessa forma, apesar da estrutura organizacional e da tomada de decisão se tornarem mais flexível e descentralizada, respectivamente, conforme sugere a teoria, os resultados empíricos não confirmam essa associação.

A teoria diz que a comunicação middle-up-down proporciona um fluxo de informações em todas as direções, resultando em maior interação se comparada as comunicações top-down e bottom-up (HEDLUND, 1994).

A análise dos dados revelou que a comunicação em todos os sentidos predomina em grande escala nos três estágios e sofre um aumento consecutivo. Corroborando esses resultados, foi identificada 
associação entre a comunicação e os estágios, portanto, a comunicação apresenta relação com o processo de desenvolvimento da GC, podendo influencia-lo, como afirma a teoria.

Diversos estudos concluem que a formalização enfraquece a GC. Quando a organização é dominada por regras formais, as novas ideias, a comunicação e a interação necessárias para criar conhecimento são restringidas. Consequentemente, a criação do conhecimento exige menor ênfase nas normas de trabalho e maior flexibilidade (LEE; CHOI, 2003).

Este estudo demonstrou que o grau de formalização das atividades aumenta com o avanço dos estágios, exatamente ao contrário do que afirma a teoria da MGC. A análise inferencial não apresentou indícios de associação entre 0 grau de formalização das atividades e o desenvolvimento das práticas de GC. Com o crescimento em tamanho e a maior complexidade há necessidade de formalizar as atividades, criar normas e regras para sistematizar e guiar os processos de GC.

A gestão do conhecimento envolve o estabelecimento de um ambiente propício à criação, transferência e uso de conhecimento (NONAKA; TAKEUCHI, 1995; DAVENPORT; DE LONG; BEERS, 1998). A organização do espaço físico deve proporcionar um ambiente que facilite a interação, a comunicação e a troca de conhecimento entre as pessoas (DAVENPORT; PRUSAK, 2003).

Apesar do ambiente permitir mais interação e troca de conhecimento entre as pessoas com 0 avanço dos estágios, principalmente no estágio 3 , quando é notável a mudança de comportamento do ambiente nas organizações estudadas, não foi possível identificar associação entre o ambiente e os estágios por meio do Teste de Fischer.

\section{Conclusão}

A presente pesquisa contribui com a literatura da área ao identificar sistematicamente os fatores que devem contemplar um MMGC. Contribui também ao explorar a relação entre um dos fatores - a infraestrutura organizacional - e os estágios da GC por meio da pesquisa empírica.

A análise descritiva dos dados possibilitou conhecer 0 comportamento da variável estudada durante o desenvolvimento da GC. A análise inferencial possibilitou identificar a existência de associação entre essas variáveis. No entanto, contrário a literatura o teste de Fischer não sugere associação entre parte dos fatores e os estágios da GC. Dessa forma, pesquisas complementares podem confirmar esse resultado e esclarecer tais causas.

As limitações do artigo dizem respeito ao fato da realização da coleta de dados ocorrer em uma única empresa. Apesar da coleta abranger diversas Unidades com características específicas e autonomia 
própria caracterizando-as cada qual como uma organização diferente; pelo fato de fazerem parte de uma mesma empresa, elas podem apresentar resultados mais homogêneos devido a alguns aspectos que possam ser semelhantes, por exemplo, a cultura da empresa. Além disso, todas elas pertencem a um único setor.

Como forma de aprofundar e complementar este estudo, pesquisas futuras podem utilizar diferentes abordagens e métodos de levantamento e análise dos dados, abordagem qualitativa e estudo de casos, por exemplo. Bem como investigar organizações de outro setor e natureza.

\section{Referências}

ADIZES, I. Os ciclos de vida das organizações: como e por que as empresas crescem e morrem e o que fazer a respeito. São Paulo: Pioneira, 1990.

BAÊTA, A.M.C. O desafio da criação. Petrópolis: Vozes, 1999.

BARUCH, Y.; HOLTOM, B.C. Survey response rate levels and trends in organizational research. Human Relations, v.61, n.8, p.1139-1160, 2008.

COLOMBO, M.G.; DELMASTRO, M. How effective are technology incubators? Evidence from Italy. Research Policy, v.31, p.1103-1122, 2002.

CONLEY, C.A.; ZHENG, W. Factors critical to knowledge management success. Advances in Developing Human Resources, v.11, n.3, p.334-348, 2009.

DAFT, R.L. Organizações: teorias e projetos. São Paulo: Pioneira Thompson Learning, 2003.

DAVENPORT, T.; DE LONG, D.; BEERS, M. Successful knowledge management projects. Sloan Management Review, v.39, n.2, p.43-57, 1998.

DAVENPORT, T.; PRUSAK, L. Conhecimento empresarial. Rio de Janeiro: Campus, 2003.

DELONG, D.W.; FAHEY, L. Diagnosing cultural barriers to knowledge management. Academy of Management Executive, v.14, n.4, p.113-127, 2000.

DRUCKER, P.F. A sociedade pós-capitalista. São Paulo: Pioneira, 1993. 
EMPRESA BRASILEIRA DE PESQUISA AGROPECUÁRIA - EMBRAPA. 2014. Disponível em: https://www.embrapa.br/. Acesso em: 01 set. 2014.

FENG, J. Constructing a knowledge management maturity model from perspective of knowledge management. In: INTERNATIONAL

ENGINEERING MANAGEMENT CONFERENCE, IEEE, 2005, p.912-917.

FENGJU, X.; XIAOJING, D. Research on the innovative enterprise knowledge management based on maturity model. In: INTERNATIONAL CONFERENCE ON PRODUCT INNOVATION MANAGEMENT, 2011, Wuhan, China, p.741-744.

FROHLICH; ROSSETTO; SILVA, 2007

GAÁL, Z. et al. Knowledge management profile maturity model. In: EUROPEAN CONFERENCE ON KNOWLEDGE MANAGEMENT, 2008, p.209 216.

GALBRAITH, J. The stages of growth. Journal of Business Strategy, v.3, n.4, p.70-79, 1982.

GOLD, A.H.; MALHOTRA, A. ; SEGARS, A.H. Knowledge management: an organizational capabilities perspective. Journal of Management Information Systems, v.18, n.1, p.185- 214, 2001.

GOMES FILHO, A. C. Inovação sistemática com responsabilidade social nos empreendimentos de base tecnológica. 2010. Tese de doutorado Universidade Federal de Santa Catarina, Florianópolis, 2010.

GRANT, R.M. Toward a knowledge-based theory of the firm. Strategic Management Journal, v.17, n.7, p.109-122, 1996.

HEDLUND, G. A model of knowledge management and the $\mathrm{N}$-form corporation. Strategic management journal, v.15, p.73-90, 1994.

INSTITUTO NACIONAL DE PROPRIEDADE INTELECTUAL - INPI. 2011. Estrutura organizacional. Disponível em: http://www.inpi.gov.br/noticias/confira-as-instituicoes-de-pesquisabrasileiras-que-mais-pedem-patentes-no-pais. Acesso em: 01 ago. 2014.

JIULING, W.; JIANKANG, W.; HONGJIANG, Y. Study on maturity level transition mechanism of knowledge management. In: INTERNATIONAL CONFERENCE ON INFORMATION MANAGEMENT, INNOVATION MANAGEMENT AND INDUSTRIAL ENGINEERING, 2012, 325-328. 
JUGEND, D.; DA SILVA, S.L. Práticas de gestão que influenciam o sucesso de novos produtos em empresas de base tecnológica. Produção, v.20, n.3, p.335-346, 2010.

KLIMKO, G. Knowledge management and maturity models, building common understanding. In: EUROPEAN CONFERENCE OF KNOWLEDGE MANAGEMENT, 2001, Kochikar, 269-278.

KRUGER, C. J.; SNYMAN, M. M. M. Formulation of a strategic knowledge management maturity model. South African Journal of Information Management, v.7, n.2, p.1-11, 2005.

KULKARNI, U.; ST LOUIS, R. Organizational self-assessment of knowledge management maturity. In: AMERICAS CONFERENCE ON INFORMATION SYSTEMS, 2003, Tampa, Florida, 2542-2551.

LEE, H.; CHOI, Y. Knowledge management enablers, process and organizational performance: an integrative view and empirical examination. Journal of Management Information Systems, v.20, n.1, p.179-228, 2003.

LEE, J.H.; KIM, Y.G.; YU, S. Stage Model for Knowledge Management. In: HAWAII INTERNATIONAL CONFERENCE ON SYSTEM SCIENCES, 2001, Maui, Hawaii.

LIN, H.F. A stage model of knowledge management: an empirical investigation of process and effectiveness. Journal of Information Science, v.33, n.6, p.643-659, 2007.

LIN, $\mathrm{H}$. Antecedents of the stage-based knowledge management evolution. Journal of Knowledge Management, v.15, n.1, p.136-155, 2011.

MARCONI, M.A.; LAKATOS, E.M. Técnicas de pesquisa. São Paulo: Atlas, 2011.

NONAKA, I.; KONNO, N. The concept of "ba": building foundation for Knowledge creation. California Management Review, v.40, n.3, p.40-54, 1998.

NONAKA, I.; TAKEUCHI, H. The knowledge-creating company. New York: Oxford University Press, 1995.

NONAKA, I.; TOYAMA, R. The knowledge-creating theory revisited: knowledge creation as a synthesizing process. Knowledge Management Research \& Practice, v.1, n.1, p. 2-10, 2003. 
O'DELL, C.; GRAYSON, C. If only we knew what we know: identification and transfer of internal best practices. California Management Review, v.40, n.3, p.154-174, 1998.

OLIVEIRA, M.; PEDRON, C.D.; MAÇADA, A.C.G. Knowledge management implementation in stages: the case of organizations in Brazil. In: EUROPEAN CONFERENCE ON KNOWLEDGE MANAGEMENT, 2010, 1-2, 752-758.

PEE, L.G.; KANKANHALLI, A. A model of knowledge organizational management maturity: based on people, process and technology. Journal of Information and Knowledge Management, v.8, n.2, p.79-99, 2009.

PENTEADO FILHO, R.C.; AVILA, A.F.D. Embrapa Brasil: análise bibliométrica dos artigos na Web of Science, 1977-2006. 2009a. Disponível em:

http://www.embrapa.br/publicacoes/tecnico/folderTextoDiscussao/arquivo s-pdf/Texto- 36_05-11-09.pdf. Acesso em: 01 nov. 2014.

PENTEADO FILHO, R.C.; AVILA, A.F.D. Estudo das citações dos artigos da Embrapa na Web of Science de 1977 a 2006. 2009b. Disponível em: http://www.embrapa.br/publicacoes/tecnico/folderTextoDiscussao/arquivo s-pdf/Texto-37_24-03-10.pdf. Acesso em: 01 out. 2014.

RASULA, J.; VUKSIAE, V.B.; STEMBERGER, M.I. The integrated knowledge management maturity model. Zagreb International Review of Economics \& Business, v.11, n.2, p.47-62, 2008.

SAVEJA, S.; JUCEVICIUS, R. The model of knowledge management system maturity and its approbation in business companies, Ocialiniai Mokslai, v.3, n.69, p.57-68, 2010.

SANTOS, I.C.; AMATO NETO, J.A. Gestão do conhecimento em indústria de alta tecnologia. Gestão e Produção, v.18, n.3, p.569-582, 2008.

SERENKO, A.; HULL, E.; BONTIS, N. An application of the knowledge management maturity model: the case of credit unions. Knowledge Management Research and Practice, in press, 2014.

SINHA, R.R.; DATE, H.A. Maturity of knowledge management and knowledge enable business process. International Journal of Research in Business and Technology, v.4, n.1, p.367-373, 2014.

TEAH; H.Y.; PEE, L.G.; KANKANHALLI, A. Development and application of a general knowledge management maturity model. In: PACIFIC ASIA CONFERENCE ON INFORMATION SYSTEMS, 2006, 1-8, 401-416. 

estágios

TEECE, D.J. Managing intellectual capital: organizational, strategic, and policy dimensions. Oxford University Press: Oxford, 2000. 